\title{
AN Q-BG-SE INTERPOLATION FOR LOW RESOLUTION IMAGES
}

\author{
Diana Earshia V \\ Research Scholar, Department of Electronics and Communication Engineering, \\ Sathyabama Institute of Science and Technology \\ Chennai, Tamilnadu 600 119, India \\ Assistant Professor, Department of Electronics and Communication Engineering, \\ Vel Tech Rangarajan Dr Sagunthala R\&D Institute of Science and Technology \\ Chennai, Tamilnadu 600062, India \\ earshy@gmail.com \\ Sumathi M \\ Professor, Department of Electronics and Communication Engineering, \\ Sathyabama Institute of Science and Technology \\ Chennai, Tamilnadu 600 119, India \\ sumagopi206@gmail.com
}

\begin{abstract}
The major threat to humankind is security. Surveillance can help for better survival. It can be done through cameras of different types. The images thus obtained may sometimes lead to Low-resolution. This is due to the following three reasons, firstly the subject of interest is in the lightly illuminated area, secondly, the distance between the subject of interest and camera is more, and thirdly the resolution of the surveillance camera is less. This article proposes a new Interpolation algorithm, Quantized Batch-Gradient sharp-Edge Interpolation algorithm (Q-BG-SE), using which the obtained low-resolution(L-R) images are transformed to high-resolution(H-R) images with much quality in image edges. This algorithm obtains appreciable objective scores as 91.4\% for SSIM, 36.505 dB for PSNR, and subjective score of 2.7831 on a scale of 3 for the full-reference image quality index (FRIQ).
\end{abstract}

Keywords: Image Interpolation; Adaptive Interpolation; Non-Adaptive Interpolation; Full reference Image Quality Index; Resampling.

\section{Introduction}

Image interpolation is a technique used to obtain new pixel values depending on the surrounding pixel value in the given image with a higher sampling rate. Interpolation is also called sampling. Thus obtained new pixel values can create unavoidable estimation errors [1]. This error will depend on two things, they are the kind of interpolation algorithm used and the prior knowledge on the original image. The computational cost, memory utilization, accurate results in terms of high-frequency components, visual quality are to be accounted for. The common artifacts found in an interpolated image are Aliasing, Smoothing, Edge Halo, Ringing, and Blocking.

\section{Related works}

Chen et al proposed enhancement of $\mathrm{HOG}$ with respect to rotation-invariant and accuracy in feature description[2]. Gradient Field HOG (GF-HOG)was proposed by Hu et al for image retrieval[3]. A Comprehensive study on non-adaptive image interpolation techniques was proposed by Diana et al[4], Loukrezis et al describe adaptive construction of sparse polynomial chaos expansions[5]. Convolution interpolation using first-order polynomials was proposed by Lin et al for real-time image reconstruction[6]. Super-resolution developed through machine learning will enhance gradient image was proposed by Noor et al [7]. A Semi-local similarity scheme for interpolating image was proposed by $\mathrm{Yu}$ et al [8]. The mismatch between a pixel of interest and residuals during parameter estimation was proposed by Hung et al[9]. Zhong et al proposed two stages in interpolating low-resolution images namely prediction algorithm followed by correction algorithm [10]. CGI's performance on the interpolated image was proposed by Ye et al [11]. This in turn reduced computation complexity. A softdecision technique used for estimating missing pixels using the piecewise autoregressive model for interpolating images was proposed by Zhang et al[12].

\section{Proposed Algorithm}

\subsection{Quantized Batch-Gradient sharp-Edge Interpolation algorithm (Q-BG-SE)}

Step 1: Obtain the Histogram of an oriented gradient of the image to be interpolated

Computes the gradients in the image to be interpolated using a Histogram of an oriented gradient. 
Step 2: Scale-up (Quantize) the gradients unto $n$ levels

The gradients are quantized into $n$ levels. The value of $n$, probably between 6 to 9 is chosen by experimentation. The pixels are grouped spatially. The gradient strengths are normalized.

Step 3: Batch up pixels with respect to the quantized intensity gradient.

Rectangular HOG blocks of size $8 \times 8$ are chosen for batch processing. The non-normalized vector $\mathrm{v}$ which consists of all histograms in a batch is normalized using L2 normalization

$$
\text { L2 normalization }=\|x\|_{2}=\left(\sum_{i}\left\|x_{i}\right\|^{2}\right)^{0.5}
$$

where $x$ is the pixel value after $\mathrm{L} 2$ normalization and $x_{i}$ is indicates nearby pixel values.

Step 4: Interpolate batches separately.

It provides Structural correlation along with four directions for improving edge information during interpolation.

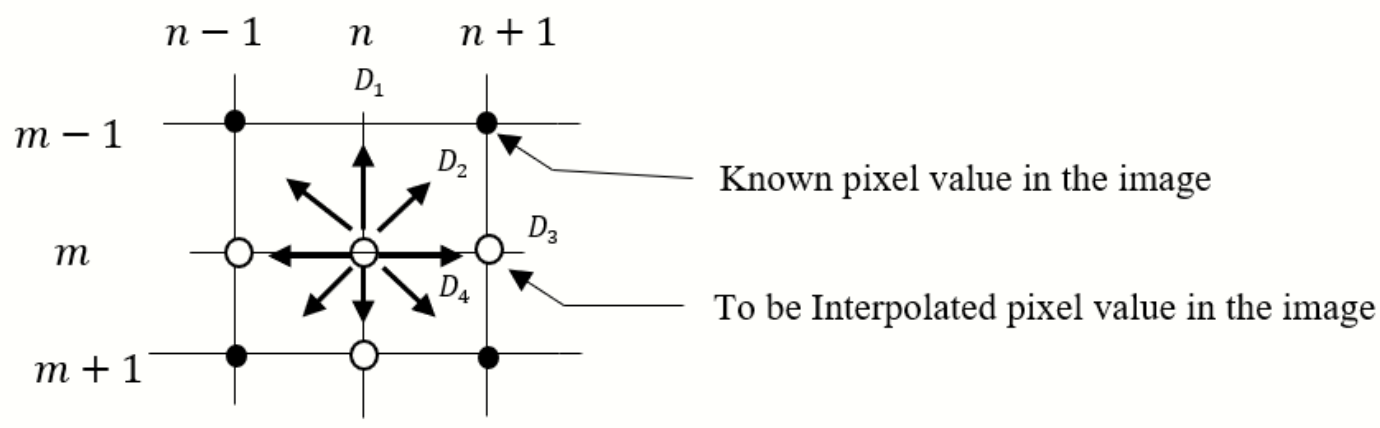

Fig. 1. Interpolation Rule for sharp Edge

Figure 1 shows the Interpolation rule for Sharp Edge. It calculates the signal variation intensity variation in four directions. The high signal variation indicates the existence of an edge perpendicular to the direction of variation[13],

$$
\begin{aligned}
& \mathrm{D} 1=\mid \begin{array}{c}
\mathrm{g}[\mathrm{m}-1, \mathrm{n}+1]+2 \mathrm{~g}[\mathrm{~m}, \mathrm{n}+1]+\mathrm{g}[\mathrm{m}+1, \mathrm{n}+1]-\mathrm{g}[\mathrm{m}-1, \mathrm{n}-1] \\
-2 \mathrm{f}[\mathrm{m}, \mathrm{n}-1]-\mathrm{f}[\mathrm{m}+1, \mathrm{n}-1]
\end{array} \\
& \mathrm{D} 2=\sqrt{2}\left|\begin{array}{c}
\mathrm{g}[\mathrm{m}-1, \mathrm{n}]+\mathrm{g}[\mathrm{m}-1, \mathrm{n}+1]+\mathrm{g}[\mathrm{m}, \mathrm{n}+1]-\mathrm{g}[\mathrm{m}, \mathrm{n}-1] \\
-\mathrm{g}[\mathrm{m}+1, \mathrm{n}-1]-\mathrm{g}[\mathrm{m}+1, \mathrm{n}]
\end{array}\right| \\
& \mathrm{D} 3=\left|\begin{array}{c}
\mathrm{g}[\mathrm{m}-1, \mathrm{n}-1]+2 \mathrm{~g}[\mathrm{~m}-1, \mathrm{n}]+\mathrm{g}[\mathrm{m}-1, \mathrm{n}+1]-\mathrm{g}[\mathrm{m}+1, \mathrm{n}-1] \\
-2 \mathrm{f}[\mathrm{m}+1, \mathrm{n}]-\mathrm{f}[\mathrm{m}+1, \mathrm{n}+1]
\end{array}\right| \\
& \mathrm{D} 4=\sqrt{2}\left|\begin{array}{c}
\mathrm{g}[\mathrm{m}, \mathrm{n}-1]+\mathrm{g}[\mathrm{m}-1, \mathrm{n}-1]+\mathrm{g}[\mathrm{m}-1, \mathrm{n}]-\mathrm{g}[\mathrm{m}, \mathrm{n}+1] \\
-\mathrm{g}[\mathrm{m}+1, \mathrm{n}+1]-\mathrm{g}[\mathrm{m}+1, \mathrm{n}]
\end{array}\right|
\end{aligned}
$$

where D1, measures gradient variation along $90^{\circ}$, D2 along $45^{\circ}$, D3 along $180^{\circ}$ and D4 along $135^{\circ}$ orientations. This is given in equations numbered between 2 to 5 .

As proposed by Cha \& Kim, the interpolated pixel should be given by

$$
g[m, n]=\frac{\sqrt{2} D_{p}}{D_{p}+\sqrt{2} D_{q}} \boldsymbol{L}_{p}+\frac{D_{p}}{D_{p}+\sqrt{2} D_{1}} L_{q}
$$

where,

$$
\begin{gathered}
D_{p}=\min (D 1, D 3), L_{p}=\min (L 1, L 3) \\
D_{q}=\min (D 2, D 4), L_{p}=\min (L 2, L 4)
\end{gathered}
$$

$\mathrm{L} 1=(\mathrm{g}[\mathrm{m}, \mathrm{n}-1]+\mathrm{g}[\mathrm{m}, \mathrm{n}+1]) / 2$

$\mathrm{L} 2=(\mathrm{g}[\mathrm{m}+1, \mathrm{n}-1]+\mathrm{g}[\mathrm{m}-1, \mathrm{n}+1]) / 2$

$\mathrm{L} 3=(\mathrm{g}[\mathrm{m}-1, \mathrm{n}]+\mathrm{g}[\mathrm{m}+1, \mathrm{n}]) / 2$

$\mathrm{L} 4=(\mathrm{g}[\mathrm{m}-1, \mathrm{n}-1]+\mathrm{g}[\mathrm{m}+1, \mathrm{n}+1]) / 2$

Consider $p=\{1,3\}$ and $q=\{2,4\}$ depending on the value of the min operation. Modifying the equation 6 by adding a regularization parameter $\lambda$. The best results are obtained experimentally by varying the value of $\lambda$ between 0.5 and 0.8 . The value of $\boldsymbol{\lambda}=\mathbf{0 . 6 5}$ yields better clarity for most of the images. 
$g[m, n]=\frac{\sqrt{2} D_{p}}{D_{p}+\sqrt{2} D_{q}} \lambda \boldsymbol{L}_{p}+\frac{D_{p}}{D_{p}+\sqrt{2} D_{1}} \lambda L_{q}$

Equation 13 gives the modified equation of 6 , for yielding better clarity in images during interpolation.

Step 5: Concatenate the batches after the application of the algorithm.

Concatenation of all the batches to obtain the final interpolated image.

Step 6: Assess the interpolated image with respect to ground truth image through objective and subjective assessments.

The quality of the interpolated image is done both in subjective and objective metrics. The objective metrics are computed using the Matlab 19a platform. It is done by comparing the Ground truth image and the Interpolated image. They are, Peak Signal to Noise Ratio (PSNR) and Structural Similarity Index (SSIM).

Equations 14 to 16 supports the computation of Peak Signal to Noise Ratio in dB.

$P S N R=10 \log _{10}\left(\frac{\left(2^{n}-1\right)^{2}}{M S E}\right)$

$M S E=\frac{1}{M * N} \sum_{m=0}^{M-1} \sum_{n=0}^{N-1} e[m, n]^{2}$

$\mathrm{e}[\mathrm{m}, \mathrm{n}]=\mathrm{y}[\mathrm{m}, \mathrm{n}]-\mathrm{g}[\mathrm{m}, \mathrm{n}]$

Equation 15 computes Mean Squares Error (MSE) in dB and Equation 16 helps to manipulate error image between ground truth image $y[\mathrm{~m}, \mathrm{n}]$ and interpolated image $\mathrm{g}[\mathrm{m}, \mathrm{n}]$.

The next metrics is the Structural Similarity Index(SSIM)[14] is calculated with the help of contrast, luminance, and structural factors between interpolated image $(\mathrm{g})$ and ground truth image (y). Equation 17 is used to manipulate SSIM. It involves parameters like mean $(\mu)$, variance $(\sigma)$, and covariance $(C)$.

$\operatorname{SSIM}(g, y)=\frac{\left(2 \mu_{g} \mu_{y}+C_{1}\right)\left(2 \sigma_{g y}+C_{2}\right)}{\left(\mu_{g}^{2}+\mu_{y}^{2}+C_{1}\right)\left(\sigma_{g}^{2}+\sigma_{y}^{2}+C_{2}\right)}$

To evaluate Mean Opinion Score (MOS) an Objective metric, 24 volunteers are involved to provide a score in a 3-scale rubric for interpolated images. This purely depends on human visual interpretation.

\subsection{Process-flow diagram}

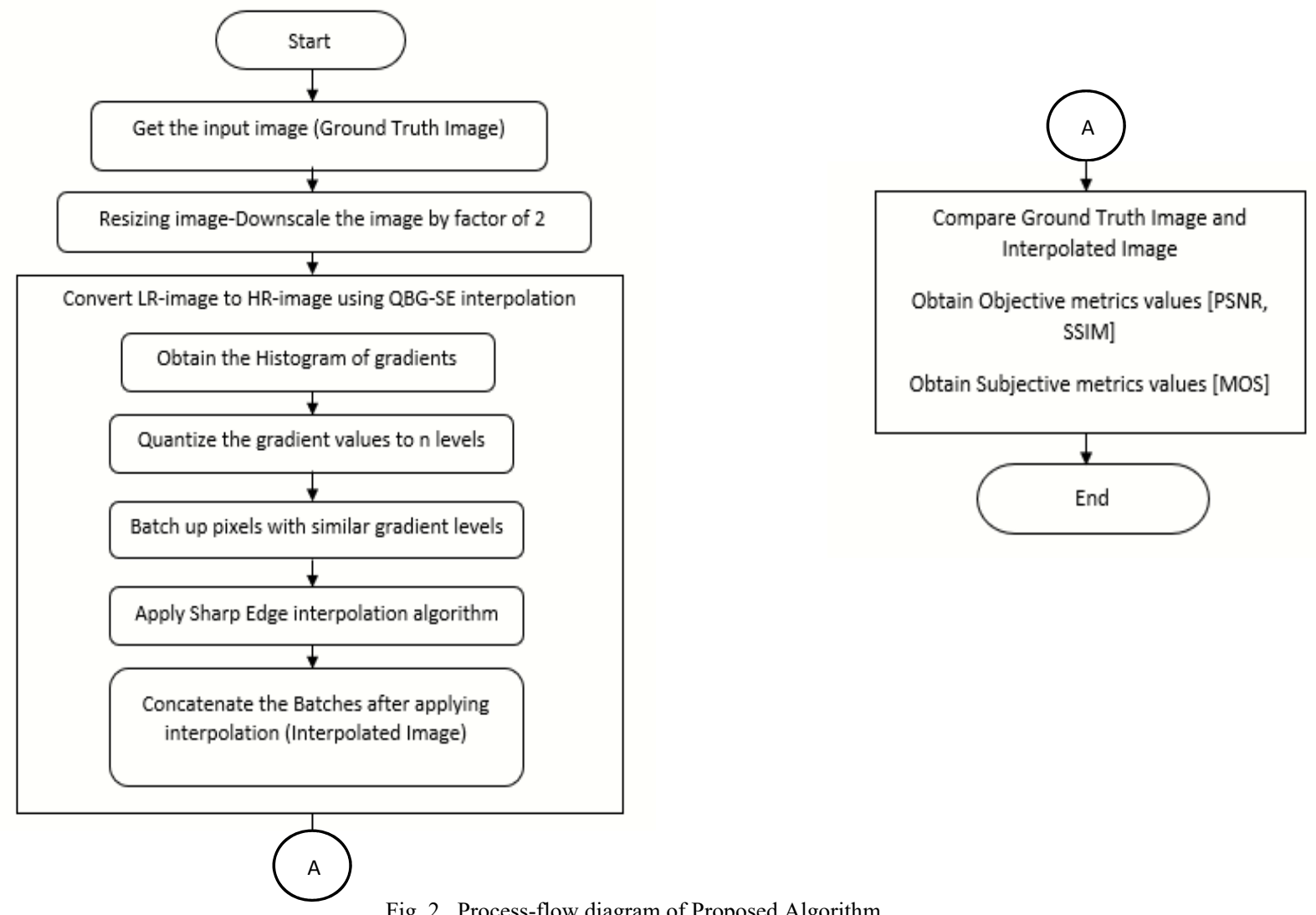

Fig. 2. Process-flow diagram of Proposed Algorithm 
Figure 2 shows the process flow of the proposed (Q-BG-SE) algorithm. The obtained input image is the ground truth image, which is downsized by a factor of $2 \times 2$. Then interpolated through the process flow involved in the proposed algorithm. Finally, the interpolated image and the ground truth image are compared to obtain Objective and Subjective metrics in order to study the performance of the proposed algorithm.

\section{Results and Discussion}

The proposed Q-BG-SE algorithm is evaluated with respect to both objective and subjective metrics. The results are compared with existing interpolation algorithms discussed in different articles.

\subsection{Experimental setup}

The code was executed on Matlab 19 platform with Intel core i5 processor. The following are test images used to analyze the proposed algorithm (Q-BG-SE) with other existing interpolation algorithms.

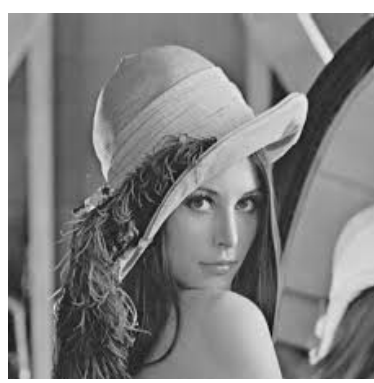

(a)

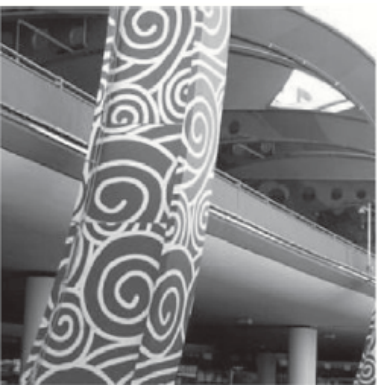

(b)

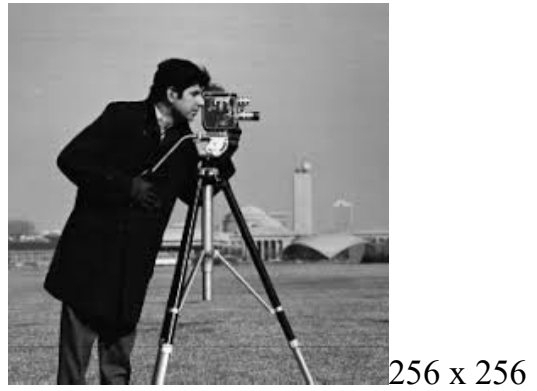

(c)

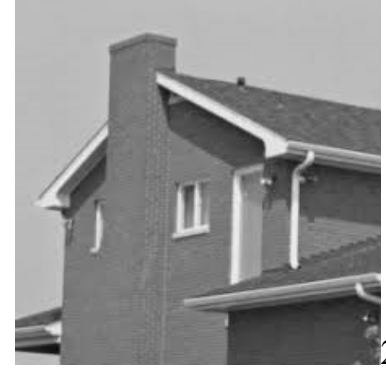

(d)
$256 \times 256$

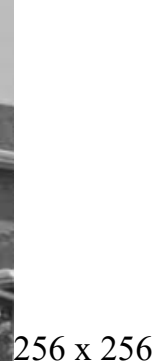

$256 \times 256$

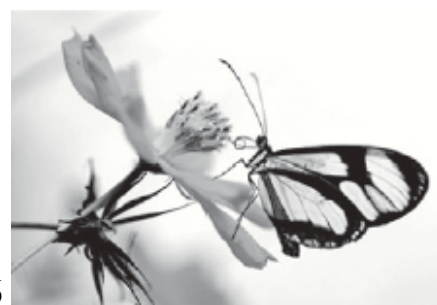

(e)

Fig. 3. (a)-(d) Test images of dimension $256 \times 256$, (e) Test image of dimension $288 \times 480$

The original images are taken as the ground truth images. These images are down-sampled by a factor of $2 \mathrm{x}$ 2 without any pre-processing. Matlab 19 is used as the software platform for evaluating the proposed algorithm (Q-BG-SE).

\subsection{Performance metrices}

\subsubsection{Objective Evaluation}

For assessing the interpolated image with respect to ground truth image the following quantitative measurement metrics (Objective evaluation) are used, which include Peak Signal to Noise Ratio (PSNR) and Structural Similarity Index (SSIM). The obtained metrics values along with other interpolation algorithms are presented in Table 1 and Table 2.

Table 1. Comparison of PSNR(dB) of various interpolation algorithm with the proposed algorithm with down-sampling by factor $2 \times 2$

\begin{tabular}{|l|c|c|c|c|c|c|}
\hline \multicolumn{1}{|c|}{$\begin{array}{c}\text { Algorithms } \\
\text { Test Images } \downarrow\end{array}$} & Bicubic[15] & NEDI[16] & NARM[12] & AGSI[17] & PCI[10] & $\begin{array}{c}\text { Q-BG-SE } \\
(\boldsymbol{\lambda}=\mathbf{0 . 6 5 )}\end{array}$ \\
\hline Lena & 30.19 & 30.57 & $\mathbf{3 1 . 3 9}$ & 31.02 & 31.18 & 31.3625 \\
\hline Station & 24.65 & 25.04 & 26.14 & 26.34 & 26.79 & $\mathbf{2 6 . 9 3 8 1}$ \\
\hline Cameraman & 25.26 & 25.38 & 25.80 & 25.29 & 26.10 & $\mathbf{2 6 . 5 4 5 2}$ \\
\hline House & 32.06 & 31.84 & $\mathbf{3 3 . 2 6}$ & 32.82 & 33.08 & $\mathbf{3 3 . 2 6 2 6}$ \\
\hline Butterfly & 26.17 & 26.88 & 27.31 & 27.67 & 27.87 & $\mathbf{2 7 . 9 1 3 8}$ \\
\hline
\end{tabular}




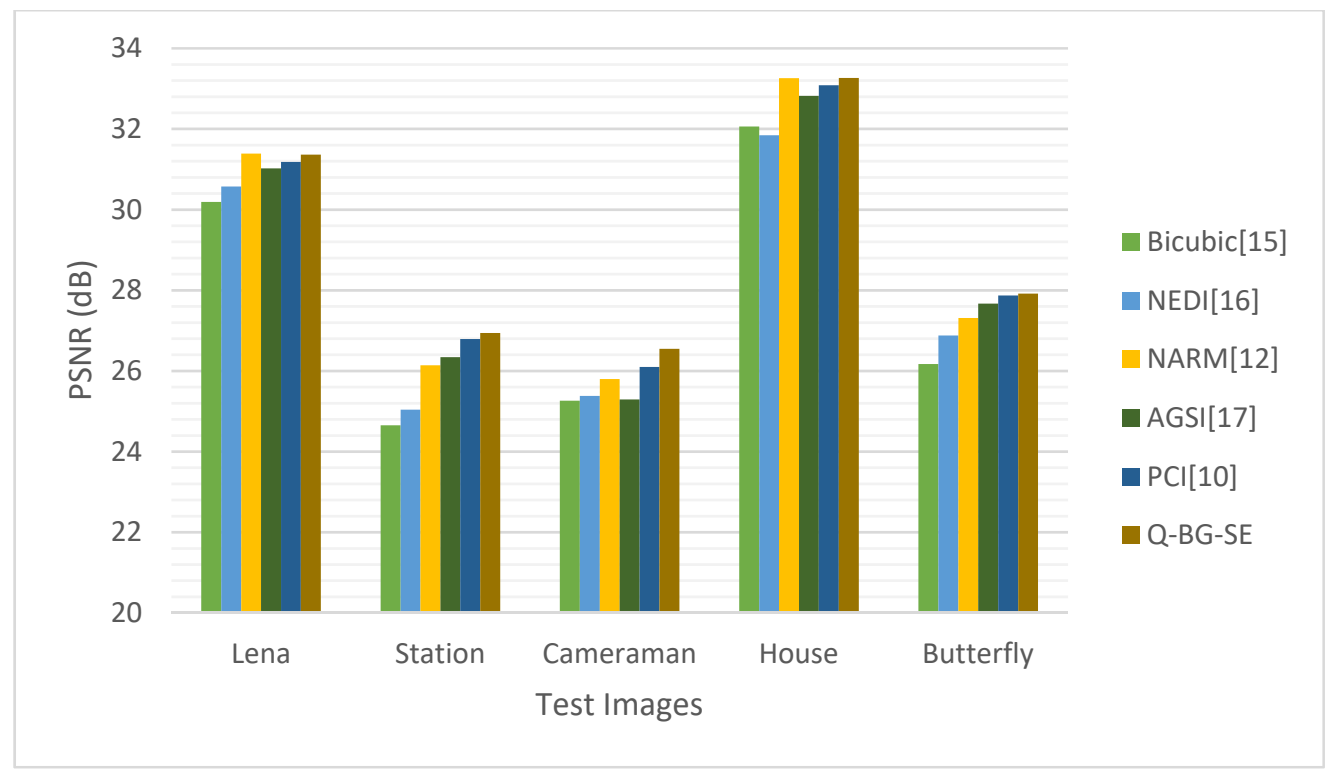

Fig. 4. Effectiveness of various algorithms with respect to PSNR(dB)

Figure 4 shows the plot of PSNR (dB) obtained when different algorithms were applied on test images. The plot shows that the proposed algorithm Q-BG-SE gives increased values of PSNR for most of the test images

Table 2. Comparison of SSIM of various interpolation algorithm with the proposed algorithm with down-sampling by factor $2 \times 2$

\begin{tabular}{|l|c|c|c|c|c|c|}
\hline \begin{tabular}{l|l} 
Algorithms \\
Test Images
\end{tabular} & \multirow{2}{*}{ Bicubic[15] } & NEDI[16] & NARM[12] & AGSI[17] & PCI[10] & $\begin{array}{c}\text { Q-BG-SE } \\
(\boldsymbol{\lambda}=\mathbf{0 . 6 5})\end{array}$ \\
\hline Lena & 0.9114 & 0.9129 & $\mathbf{0 . 9 2 4 4}$ & 0.9178 & 0.9211 & 0.9234 \\
\hline Station & 0.8928 & 0.9023 & 0.9214 & 0.9215 & 0.9286 & $\mathbf{0 . 9 2 9 8}$ \\
\hline Cameraman & 0.8649 & 0.8647 & $\mathbf{0 . 8 7 7 9}$ & 0.8690 & 0.8746 & 0.8776 \\
\hline House & 0.8747 & 0.8721 & 0.8821 & 0.8793 & 0.8787 & $\mathbf{0 . 8 7 9 6}$ \\
\hline Butterfly & 0.9507 & 0.9562 & $\mathbf{0 . 9 6 3 5}$ & 0.9626 & $\mathbf{0 . 9 6 3 5}$ & $\mathbf{0 . 9 6 3 5}$ \\
\hline
\end{tabular}

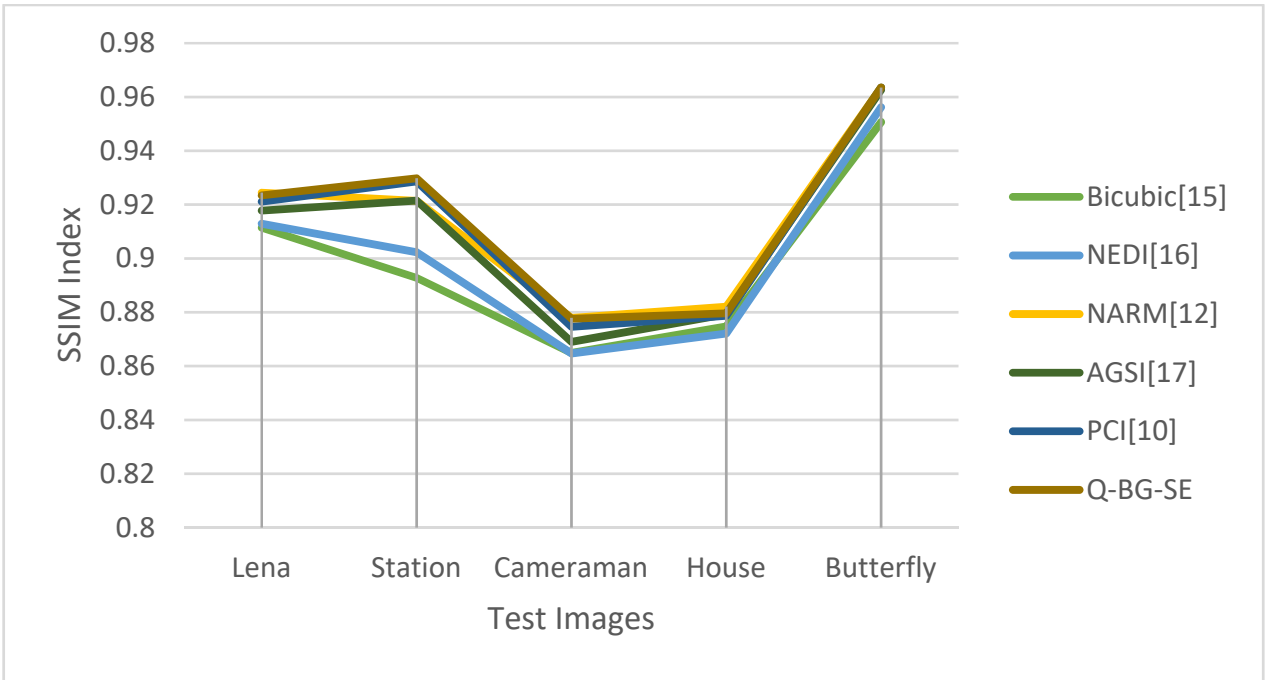

Fig. 5. Similarity Index of interpolated images with ground truth images

From fig. 5 it can be observed that Q-BG-SE algorithm obtains on an average 0.91478 (i.e) $91.4 \%$ of similarity of ground truth images, when compared to other algorithms such as 89.89 for Bicubic, 90.16 for NEDI, 91.38 for NARM, 91.0 for AGSI, 91.33 for PCI. 


\subsubsection{Subjective Evaluation}

For subjective evaluation of interpolated images Mean opinion score (MOS) is used. This metrics purely depends on Human Visual Interpretation. A set of 24 people with good vision are selected to compare the interpolated image with the ground truth image on 3-scale rubrics, where 1 is worst, 2 is better, 3 is best. These people are to provide score based on the quality and clarity of interpolated images. Finally, the mean of the obtained scores including all the samples of images are calculated for comparison.

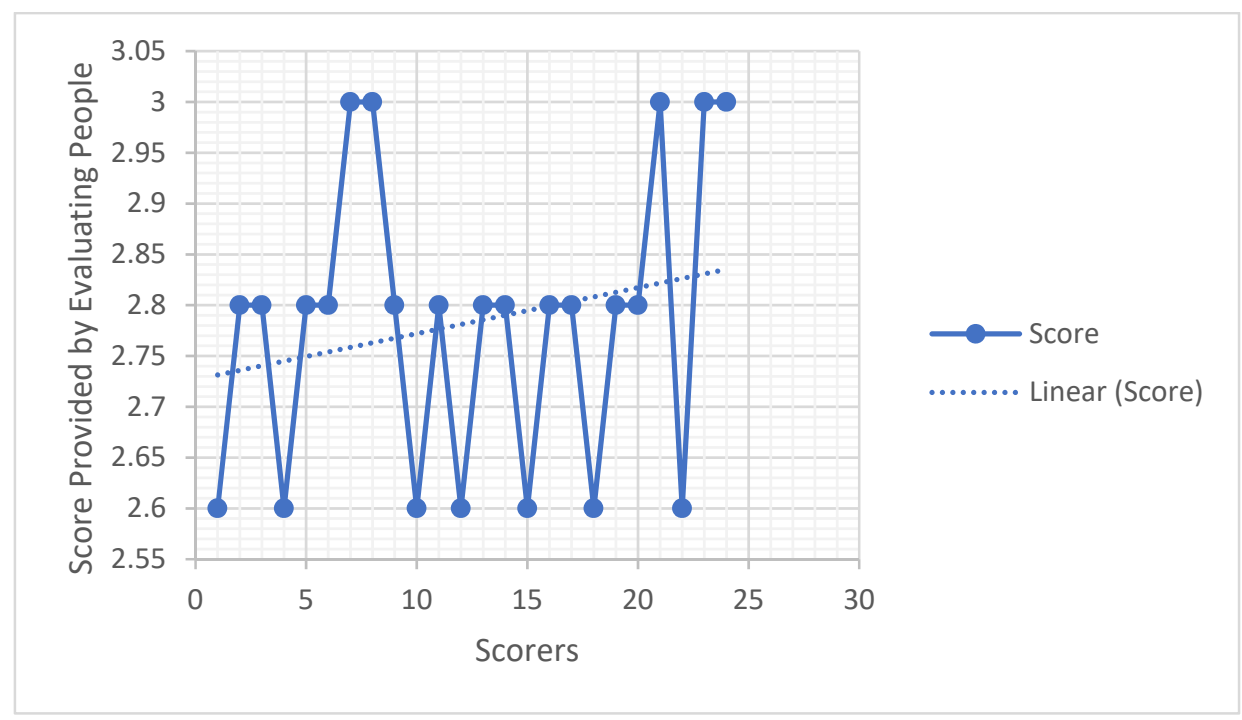

Fig. 6. Calculation of Mean opinion Score (MOS) using Q-BG-SE algorithm for test images (a) - (e)

Figure 6 shows the plot of score with scorer MOS calculation. Table 3 shows the comparison of MOS scores between PCI and Q-BG-SE algorithms.

Table 3. comparison of Mean Opinion Score (MOS) between PCI and Q-BG-SE (proposed) algorithms

\begin{tabular}{|c|c|c|}
\hline \multirow{2}{*}{ Mean Opinion Score } & PCI[[10]] & Q-BG-SE $(\boldsymbol{\lambda}=\mathbf{0 . 6 5})$ \\
\cline { 2 - 3 } & 2.776 & $\mathbf{2 . 7 8 3 1}$ \\
\hline
\end{tabular}

The below mentioned Table 5 shows the concatenation of Ground truth, Down-sampled and interpolated images with Histogram of Oriented Gradient and its Structural Similarity Index (SSIM) obtained after applying the proposed (Q-BG-SE) algorithm.

Table 5. Concatenation of Ground truth, Down-sampled and Interpolated images with SSIM

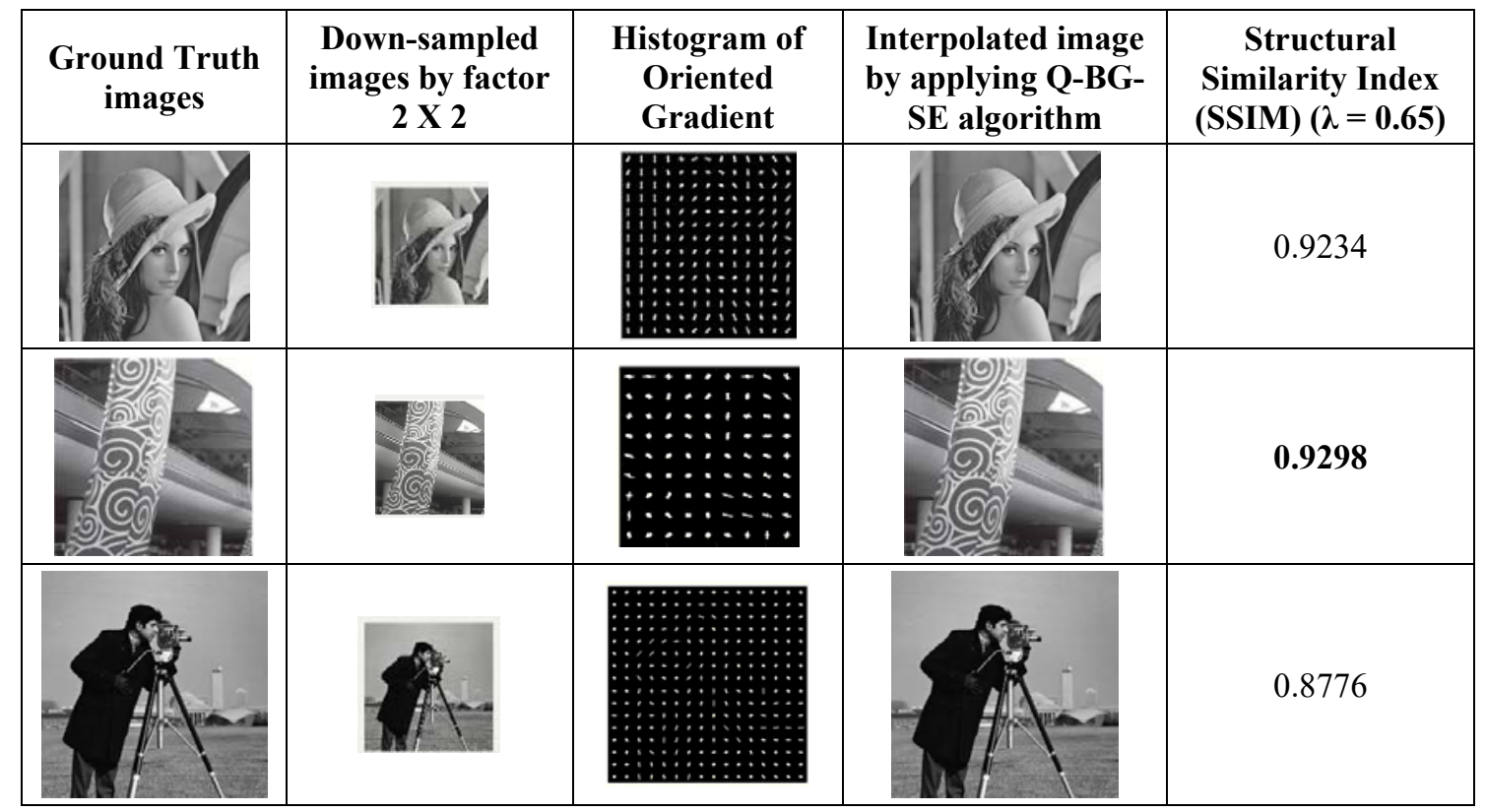




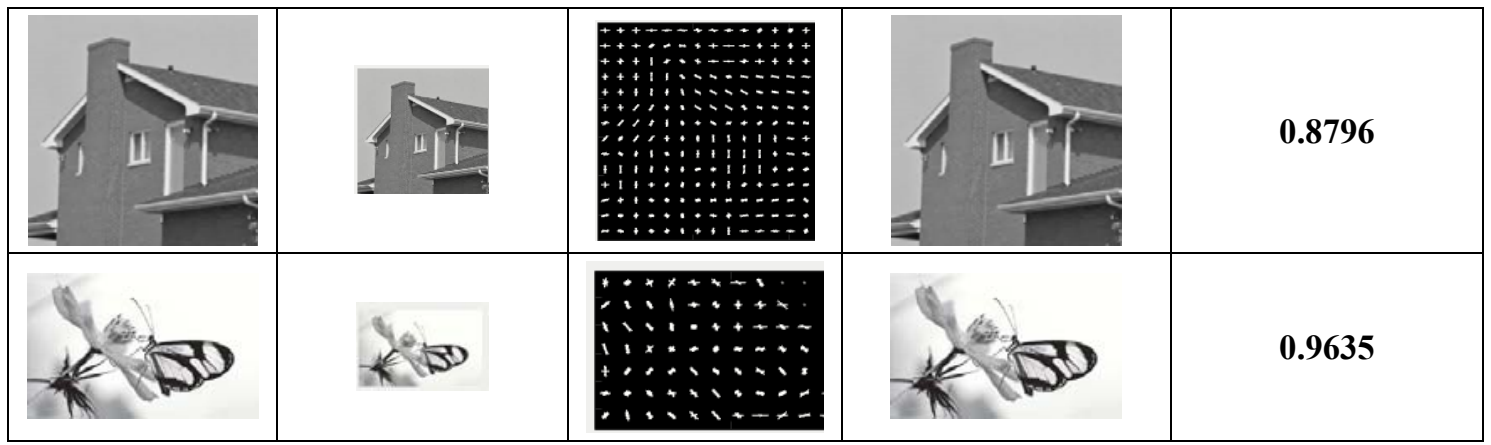

\section{Conclusion}

The obtained results of Objective and Subjective metrics prove that the proposed, Quantized Batch-Gradient Sharp-Edge (Q-BG-SE) algorithm yields competitive quality of interpolated images with respect to existing algorithms. Two different sizes of images ( $256 \times 256$ and $288 \times 480)$ were used for experimentation. The structural similarity Index (SSIM) and Peak Signal to Noise Ratio (PSNR) were found to be $91.4 \%$ and $36.505 \mathrm{~dB}$ on an average for the test images. Therefore, the poor resolution surveillance images can be interpolated to obtain highresolution images, which in-turn helps in securing human lives.

\section{References}

[1] C.-W. Kok and W.-S. Tam, "Nonadaptive Interpolation,” Digit. Image Interpolat. MATLAB®, pp. 91-122, 2018.

[2] J. Chen, T. Nakashika, T. Takiguchi, and Y. Ariki, "Content-based image retrieval using rotation-invariant histograms of oriented gradients,” ICMR 2015 - Proc. 2015 ACM Int. Conf. Multimed. Retr., pp. 443-446, 2015.

[3] R. Hu and J. Collornosse, "A performance evaluation of gradient field HOG descriptor for sketch based image retrieval," Comput. Vis. Image Underst., vol. 117, no. 7, pp. 790-806, 2013.

[4] M. S. V.Diana Earshia, A Comprehensive Study of 1D and 2D Image Interpolation Techniques, vol. 500, no. september. Springer Singapore, 2018.

[5] D. Loukrezis and H. De Gersem, “Adaptive Sparse Polynomial Chaos Expansions via Leja Interpolation,” pp. 1-22, 2019.

[6] C. C. Lin, M. H. Sheu, C. Liaw, and H. K. Chiang, "Fast first-order polynomials convolution interpolation for real-time digital image reconstruction," IEEE Trans. Circuits Syst. Video Technol., vol. 20, no. 9, pp. 1260-1264, 2010.

[7] D. F. Noor, Y. Li, Z. Li, S. Bhattacharyya, and G. York, "Multi-Scale Gradient Image Super-Resolution for Preserving SIFT Key Points in Low-Resolution Images," Signal Process. Image Commun., vol. 78, no. November 2018, pp. 236-245, 2019.

[8] L. Yu and M. T. Orchard, "Single Image Interpolation Exploiting Semi-local Similarity," ICASSP, IEEE Int. Conf. Acoust. Speech Signal Process. - Proc., vol. 2019-May, pp. 1722-1726, 2019.

[9] K. W. Hung and W. C. Siu, "Robust soft-decision interpolation using weighted least squares," IEEE Trans. Image Process., vol. 21 , no. 3, pp. 1061-1069, 2012.

[10] B. Zhong, K. K. Ma, and Z. Lu, “Predictor-corrector image interpolation,” J. Vis. Commun. Image Represent., vol. 61, pp. 50-60, 2019.

[11] W. Ye and K. K. Ma, “Convolutional Edge Diffusion for Fast Contrast-guided Image Interpolation,” IEEE Signal Process. Lett., vol. 23, no. 9 , pp. 1260-1264, 2016.

[12] X. Zhang and X. Wu, "Image interpolation by adaptive 2-D autoregressive modeling and soft-decision estimation," IEEE Trans. Image Process., vol. 17, no. 6, pp. 887-896, 2008.

[13] Y. Cha and S. Kim, "The error-amended sharp edge (EASE) scheme for image zooming," IEEE Trans. Image Process., vol. 16, no. 6, pp. 1496-1505, 2007.

[14] Z. Wang, A. C. Bovik, H. R. Sheikh, and E. P. Simoncelli, "Image quality assessment: From error visibility to structural similarity," IEEE Trans. Image Process., vol. 13, no. 4, pp. 600-612, 2004.

[15] R. G. Keys, “Cubic Convolution Interpolation for Digital Image Processing,” IEEE Trans. Acoust. Speech Signal Process., vol. ASSP29, no. 6, p. $1153,1981$.

[16] X. Li and M. T. Orchard, "New edge-directed interpolation,” IEEE Trans. Image Process., vol. 10, no. 10, pp. 1521-1527, 2001.

[17] M. Li, J. Liu, J. Ren, and Z. Guo, “Adaptive General Scale Interpolation Basedon Weighted Autoregressive Models,” vol. 25, no. 2, pp. $200-211,2015$ 